Recepción: 15/01/2019

Aceptación: 19/03/2019

Publicación: 05/05/2019

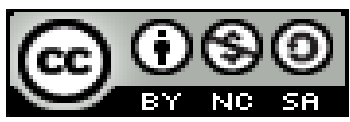

Ciencias económicas y empresariales

Artículo de revisión

\title{
Estudio documental sobre los ámbitos de investigación de la carrera de administración de empresas "organización y estrategia empresarial"
}

\section{Analysis of the factors associated with the sustainability of the enterprises in the planning area 7 - South of Ecuador}

\section{Análise dos fatores associados à sustentabilidade dos empreendimentos naárea de planejamento 7 - Sul do Equador}

Jorge Edwin Ormaza-Andrade ${ }^{\mathrm{I}}$
jormaza@ucacue.edu.ec

Jorge Oswaldo Quevedo-Vázquez II

joquevedov@ucacue.edu.ec

Manuel Rafael Quevedo-Barros III

mrquevedob@ucacue.edu.ec

\author{
Ramiro Oswaldo González-Rodríguez IV \\ rogonzalezr@ucacue.edu.ec
}

Rolando Patrício Andrade-Amoroso v
randradea@ucacue.edu.ec

Correspondencia: jormaza@ucacue.edu.ec

\footnotetext{
${ }^{\text {I }}$ Magíster en Administración de Empresas Mención en Recursos Humanos y Marketing, Especialista en Docencia Universitaria, Ingeniero Empresarial, Contador Público, Auditor, Docente de la Universidad Católica de Cuenca Sede Azogues Unidad Académica de Administración Facultad de Administración de Empresas, Azogues, Ecuador.

II Magíster en Administración de Negocios, Diploma Superior en Práctica Docente Universitaria, Economista, Licenciado en Economía y Finanzas, Subdirección de Posgrados Universidad Católica de Cuenca. Unidad Académica de Administración Facultad de Administración de Empresas, Azogues, Ecuador.

III Magíster en Contabilidad y Auditoría, Licenciado en Administración, Contador Público, Docente de la Universidad Católica de Cuenca Sede Azogues, Unidad Académica de Administración, Facultad de Contabilidad y Auditoría, Cuenca. Ecuador.

IV Magíster en Administración de Empresas Mención en Recursos Humanos y Marketing, Ingeniero Empresarial, Auditor, Contador Público, Docente de la Universidad Católica de Cuenca Sede Azogues Unidad Académica de Administración Facultad de Contabilidad y Auditoría. Azogues, Ecuador.

${ }^{v}$ Magíster en Administración de Empresas Mención en Recursos Humanos y Marketing, Ingeniero Empresarial, Auditor, Contador Público, Docente de la Universidad Católica de Cuenca. Unidad Académica de Administración Facultad de Contabilidad y Auditoría. Cuenca, Ecuador.
} 


\title{
Resumen
}

Este artículo presenta un estudio documental acerca de cómo los autores han abordado la investigación de temas referidos a la administración de empresas y más precisamente a la organización y a la estrategia empresarial. Se han revisado textos, libros, artículos y trabajos de autores como Romero, (2002), Lozada y Gutiérrez, (2010), Suarez e Ibarra, (2008), Koot \& Weinth, (2010), Aragón, Rubio, Serna y Chablé, (2010) González, Aguilera y Rodríguez, (2011), entre otros. Desde el punto de vista metodológico se ha asumido esta investigación como documental, la misma se adopta como una investigación confirmatoria de diseño documental, según Hurtado de B., (1998) en Fernández, (2007), así también se corresponde con una investigación de tipo descriptiva según Hernández, Fernández y Baptista (2014). De la revisión se obtuvo que las estrategias son acciones que se adoptan y efectúan en el desarrollo de una actividad empresarial con el fin de obtener resultados positivos, teniendo guías en un grupo para desempeñar las diferentes actividades con liderazgo del gerente. Entre las conclusiones se tiene la siguiente: La estrategia es el camino que las organizaciones siguen para alcanzar un objetivo, para mejorar el desempeño dentro de la organización, mientras que el proceso de toma de decisiones estratégicas es el que sirve para corregir el rumbo de las organizaciones, hacia un objetivo más deseable.

Palabras Clave: Estudio documental; administración de empresas; organización y estrategias empresariales.

\begin{abstract}
This article presents a documentary study about how the authors have approached the research of topics related to the administration of companies and more precisely to the organization and the business strategy. Texts, books, articles and works by authors such as Romero, (2002), Lozada \& Gutiérrez, (2010), Chavelino, (2012), Suarez \& Ibarra, (2008), Koot \& Weinth, (2010), Aragón have been revised, Rubio, Serna \& Chablé, (2010) González, Aguilera \& Rodríguez, (2011), among others. From the methodological point of view, this research has been assumed as a documentary, it is assumed as a confirmatory documentary design research, according to Hurtado de B., (1998) in Fernández, (2007), this is also the case with an investigation of descriptive type
\end{abstract}


according to Hernández, Fernández and Baptista (2014). From the review it was obtained that the strategies are actions that are adopted and carried out in the development of a business activity in order to obtain positive results, having guides in a group to carry out the different activities with leadership of the manager. Among the conclusions is the following: The strategy is the path that organizations follow to reach an objective, to improve the performance within the organization, while the process of strategic decision making is the one that serves to correct the course of the organizations, towards a more desirable goal.

Keywords: Documentary study, business administration, organization and business strategies.

\section{Resumo}

Este artigo apresenta um estudo documental de questões de investigação como os autores abordados relacionados à administração de empresas e mais precisamente para a estratégia da organização e de negócios. Eles foram revistos textos, livros, artigos e obras de autores como Romero (2002), Lozada e Gutierrez (2010), Suarez e Ibarra (2008), Koot \& Weinth, (2010), Aragão, Rubio, Serna e chable (2010) González, Aguilera e Rodríguez, (2011), entre outros. Do ponto de vista metodológico foi tomada sobre esta investigação como um documentário, ele é adotado como um projeto de pesquisa documental de confirmação, segundo Hurtado de B., (1998), Fernandez (2007) e corresponde também a uma investigação tipo descritivo segundo Hernández, Fernández e Baptista (2014). A revisão foi obtido que as estratégias são ações que são tomadas e feitas no desenvolvimento de uma atividade empresarial, a fim de alcançar resultados positivos, com guias em um grupo para realizar atividades diferentes com o gerente liderança. Entre as conclusões, é a seguinte: A estratégia é a maneira que as organizações seguir para alcançar um objetivo, para melhorar o desempenho dentro da organização, enquanto o processo de tomada de decisões estratégicas é usado para corrigir o curso da organizações, para um objetivo mais desejável.

Palavras Chave: Estudo documental; administração de empresas; organização e estratégias de negócios. 


\section{Introducción}

Las Ciencias de la Administración implican el estudio del comportamiento humano en el sentido de su gestión administrativa, en correspondencia con el alcance de metas, objetivos, la misión y visión de una organización empresarial dada. Todo ello se corresponde con el hecho de que, el éxito de una empresa depende de la gerencia y el liderazgo de los responsables del talento humano que se desempeñan en la organización. En ese sentido, es menester conocer y analizar las tendencias de los autores latinoamericanos en cuanto a estos temas.

En ese orden, se considera que la acción empresarial permite el alcance de metas y objetivos previamente planificados, por lo que se requiere determinar los objetivos de la organización, en correspondencia con la misión y la visión de una determinada empresa. Por tanto, se concuerda con Arce, (2010): quien apunta que "la acción empresarial debe contener en sí misma una finalidad, la que, a su vez dota de misión a la organización en cuestión, cuyos gerentes determinarían las estrategias a seguir en correspondencias con sus objetivos y metas·. Es así como se considera que, las estrategias se definen como los medios que se emplean para alcanzar los objetivos planteados de una compañía dada, lo que representa el más alto nivel de la gerencia. Todo ello se configura en sendos procesos metodológicos para la administración de los recursos materiales y la coordinación del talento humano que labora en las organizaciones.

Desde este marco contextual, en este artículo se presenta una revisión y análisis documental de artículos y textos de autores de diversas latitudes, haciendo énfasis en autores latinoamericanos que han abordado el estudio de las ciencias administrativas con especial interés en las estrategias gerenciales para la resolución de asuntos administrativos. Las estrategias gerenciales se definen como acciones de gestión que implican el alcance de un significativo nivel de competitividad, lo cual es fundamental en los procesos de gestión de una organización, lo cual es importante porque coadyuva hacia el establecimiento de una buena organización empresarial. En ese tenor, la estrategia se asume como una herramienta esencial en los procesos administrativos y el manejo general de una empresa determinada.

Su capital importancia radica en el hecho de la que las estrategias gerenciales podrían impulsar el alcance de la productividad y la competitividad de las empresas. Todo ello comporta el hecho en

\section{4}

Pol. Con. (Edición núm. 33) Vol. 4, No 5, mayo 2019, pp. 411-430, ISSN: 2550 - 682X 
sí mismo de que, los recursos, los bienes materiales y el talento humano que trabaja en las organizaciones deben demostrar fidelidad a las metas y los objetivos institucionales de la organización en cuestión, en pos de lograr el éxito económico y el desarrollo empresarial, hacia la mejora de la calidad de la productividad de la empresa, con arreglo a valores como competitividad y rendimiento económico. Desde este marco contextual se declara que, en este artículo se presenta una revisión documental de artículos de autores latinoamericanos que han permitido la derivación de una serie de unidades de análisis, categorías y subcategorías que coadyuvarían a la derivación posterior, de sendos soportes teóricos que explican el complejo mundo empresarial, en términos de estrategias gerenciales para la competitividad y el rendimiento laboral, económico y financiero de las organizaciones empresariales en general.

\section{Materiales y Métodos}

En este apartado se insertan los aportes teóricos esenciales sobre la actividad gerencial, la estrategia empresarial, las organizaciones desde un enfoque tecnológico sustentadas en la innovación y el uso de las Tic, la adopción de la estrategia como un mecanismo que apunta hacia el logro de la productividad y la competitividad en las empresas en pos de alcanzar el éxito económico, la efectividad de los procesos, el crecimiento y el desarrollo de la organización.

\section{Referentes teóricos que sustentan la estrategia gerencial. Una revisión documental}

Toda actividad empresarial comporta el alcance de metas y objetivos previamente planificados, por lo que es fundamental el establecimiento de fines económicos, en correspondencia con la misión y la visión de una determinada empresa. Tan es así que, en palabras de Arce, (2010): se establece que la acción empresarial debe contener en sí misma una finalidad, la que, a su vez dota de misión a la organización en cuestión, cuyos gerentes determinarían las estrategias a seguir en correspondencias con sus objetivos y metas. De esta manera, las estrategias se asumen como los medios que se emplean para alcanzar los objetivos planteados de una compañía dada, lo que representa el más alto nivel de la gerencia. Convirtiéndose en un método de uso y de asignación de los recursos disponibles dentro de una organización, con el fin de que la empresa pueda alcanzar un equilibrio competitivo dentro del mercado en el que opera. También se acota que mientras una empresa posea mayor información, ésta podría disponer de la formulación de estrategias mucho más amplias. 
En ese orden, se adopta la perspectiva apuntada por Romero, (2002), quien aduce que:

Las organizaciones de la nueva eran, también llamada "era de la información”, han conocido el verdadero poder de la tecnología, no sólo por su capacidad de hacer que una empresa sea más eficiente, sino también su potencial para desatar un cambio completo. (p. 22)

Al hacer alusión a este tema se refiere que, para alcanzar el éxito en las organizaciones de este siglo se requiere el uso de tecnología e innovación, que han venido sustituyendo en algunos casos, a la fuerza laboral; esto trae como consecuencia una tasa de desempleo alta en la economía de un país, además se relaciona con nuevas formas de interacción y el uso efectivo de la tecnología dentro de las organizaciones, que provoca que la fuerza humana sea paulatinamente sustituida. De allí que se haga preciso la inclusión de estrategias y el uso de competencias estratégicas por parte de los gerentes y de los trabajadores en general. De tal suerte, lo expresan los autores Lozada, Gutiérrez y Arias (2010) al afirmar lo que sigue a continuación:

El campo de estudio de la estrategia se ha consolidado como uno de los más representativos, por lo cual académicos y. gerentes consideran la estrategia como herramienta que cobra especial importancia en la búsqueda de ventaja competitiva y soporte esencial. Desde la década de los sesenta la estrategia ha logrado posicionarse como uno de los principales elementos que caracterizan la capacidad de dimensionar la reflexión y posterior acción racional de la organización. Para el desarrollo de la capacidad estratégica de quienes se forman en los programas académicos de administración y que tienen a su cargo la gestión de las empresas, adquiere especial relevancia el contexto local en el desarrollo y consolidación de capacidades organizacionales. (p. 39)

Al interpretar esta cita, se infiere que la estrategia se considera como una herramienta primordial que busca una ventaja competitiva, un soporte esencial para la gestión de organización, lo cual es relevante, ya que ayuda al establecimiento de una buena organización y el control para sacar 
adelante a la empresa. En ese orden, desde la década de las sesenta la estrategia se ha convertido en un elemento muy importante para el buen manejo de las empresas. De modo que se pueda alcanzar una ventaja realmente competitiva. Esto quiere decir que, todos los que trabajan en las empresas deben tener una alta capacidad de organización para conseguir un excelente desarrollo empresarial, en pos de mejorar la calidad de la empresa y tener mayores ventas en el país.

En palabras de los autores Cantero y Leyva, (2016) las empresas han surgido al tiempo que van desarrollando capacidad para adaptarse y satisfacer las demandas de la población que atiende a la vez que se adapta al contexto en el que se circunscribe. Teniendo en cuenta lo antes expuesto, "las empresas han de encaminarse a idear alternativas, buscar formas de financiamiento, reducir gastos, reordenar procesos, con el propósito de optimizar recursos para incrementar al máximo la eficiencia, para alcanzar los resultados propuestos y llevar su economía al camino del éxito".

En ese marco, desde la revisión realizada en el sistema empresarial cubano se observó que el mismo está sometido constantemente a nuevas propuestas de mejora con el objetivo de lograr la optimización de su economía, debido a que en su mayoría son organizaciones con fines de lucro, por lo que el logro de ingresos monetarios constituye el pilar fundamental. Dentro de este grupo de organizaciones, se destacan las empresas de servicio, cuyo objeto social es ofrecer un bien tangible o intangible con la calidad y efectividad requeridas, y con ello brindar satisfacción material al consumidor.

Por su parte, desde el punto de vista de los autores Corrêa, Sugahara y Rodríguez, (2015) se considera que: 'Para una organización, innovar implica asumir cambios o contribuir para que estos sean posibles en su respectivo entorno de negocios lo que puede influir significativamente en su competitividad". Por lo que se puede deducir que toda organización que tenga como objetivo ser competitiva debe contemplar la innovación como una de sus estrategias o, por lo menos, adoptar estrategias que conduzcan hacia la innovación, ya que es por medio de ésta que la empresa modifica los cambios en el mercado.

Aunado a ello, Chávez (2012) explica que: 'Las empresas que hayan aprendido a evaluar y a desarrollar las competencias de su personal estarán más capacitadas para afrontar los continuos retos de su entorno". Por lo que, desde estas consideraciones, en este artículo se realiza un análisis documental sobre la estrategia interna que pueden realizar los líderes de una 
organización; aprovechar el conocimiento, habilidades y capacidades de sus colaboradores para mejorar el desempeño dentro de la organización. De esta manera, lograr cumplir con las exigencias del entorno donde se encuentra y poder lograr los objetivos propuestos.

A la luz de estas consideraciones, se asume que: La productividad, la competitividad y la perdurabilidad deben configurarse en objetivos de la gerencia desde una gestión que se distinga por procesos de calidad total, reingeniería, gestión del cambio, teoría de las restricciones y la aplicación de sistemas de control interno. Estas herramientas generan resultados positivos que se traducen en una gestión efectiva y productiva; sin embargo, en algunos casos no se logra el resultado esperado.

En ese orden, es consideración de los autores Leal y Cefalá, (2003: 41), que:

Cuando la estrategia no está dando respuesta a las necesidades actuales de la organización, existe una inadecuada relación entre las respuestas teóricas sobre estrategia, los modelos que se proponen y las necesidades reales de los operadores estratégicos a la hora de adoptar elecciones para enfrentarse a los nuevos retos. Por tanto, se hace necesario la búsqueda de nuevas vías para comprender la estrategia.

También se puede decir que las organizaciones se desenvuelven actualmente en un medio cambiante; caracterizado por avances tecnológicos, transformaciones políticas, sociales y económicas, que producen modificaciones en sus estructuras organizativas y exigen la reformulación de sus estrategias gerenciales, como manera de responder a las exigencias de un entorno cada vez más competitivo, incierto y globalizado. El sector de la distribución de bienes de consumo masivo ha sido uno de los sectores afectados por esos procesos de cambio y es el que ha respondido más rápidamente ante los mismos. En otros casos, se puede decir que es un proceso continuo de toma de decisiones estratégicas que no se preocupa por anticipar decisiones que deban tomarse en el futuro, sino por considerar las implicaciones futuras de las decisiones que deban tomarse en el presente. En ese orden Suárez e Ibarra, (2008) expresan que:

En toda empresa existe una jerarquía de objetivos: cada objetivo se fija teniendo cuenta una orientación más amplia o tomando como 
referencia objetivos más amplios de la organización para evitar la dispersión de esfuerzos o pérdida de la unión organizacional de la empresa.

En ese sentido puede afirmarse que, mediante el análisis ambiental la empresa procura conocer su ambiente externo y diagnosticar qué ocurre en él. Para que la empresa pueda operar con eficiencia y eficacia, es necesario que conozca el ambiente externo que le rodea: sus necesidades, oportunidades, recursos disponibles, dificultades y restricciones, amenazas, coacciones y contingencias de las que no puede huir ni escapar. Dado que el ambiente cambia continuamente, el nivel institucional de la empresa debe analizar sistemáticamente y diagnosticar las condiciones ambientales que rodean la empresa.

El análisis ambiental puede llevarse a cabo con base en información verbal o escrita, investigación y análisis, espionaje, estudios de previsión y sistemas de información gerencial, que varían de acuerdo con la empresa. La formulación de las alternativas estratégicas puede llevarse a cabo dentro de estándares rutinarios o bastante creativos. Dependiendo de la situación externa y la interna, puede darse preferencia a estrategias activas o pasivas. Las grandes empresas dominantes desarrollan estrategias ofensivas en sus principales segmentos de mercado, mientras que las pequeñas prefieren sobrevivir mediante estrategias defensivas cuando actúan en mercados copados por las grandes empresas, o mediante estrategias ofensivas en segmentos de mercado ignorados por las empresas dominantes. Las empresas pueden combinar estrategias ofensivas (activas) y estrategias defensivas (pasivas) en diversas áreas del ambiente de tarea.

En esa línea de pensamiento, autores como Koot \& Weinth, (2010) manifiestan que: 'La estrategia empresarial es lo que hace que toda empresa represente más que la suma de sus partes". El mundo empresarial global está caracterizado por las apreciaciones económicas de rentabilidad extrema, recuperación del capital de inversión en el menor tiempo, pasando por alto todos los lineamientos de gestión empresarial disponibles. Es por ello que, se pueden observar ciclos irregulares de inversión, focalización pasajera de beneficios sin proyección de largo plazo, movimientos financieros que desbordan la calidad de la inversión, análisis corto plazo de riesgo, etc.

En ese tenor, los autores Aragón, Rubio, Serna y Chablé, (2010) manifiestan que: 
Este proceso permite afirmar que el éxito competitivo en las MiPyMEs (Micro, Pequeñas y Medianas Empresas) está influenciado positivamente por la innovación, tecnología, calidad del producto o servicio, adecuada dirección gestión de los recursos humanos, la capacidad directiva y el uso de estrategias competitivas. (p. 27)

Desde esta dirección, se propone un modelo explicativo del éxito competitivo o competitividad que integra los dos enfoques teóricos indicados: la influencia en el éxito competitivo de la estrategia empresarial y confirmar la influencia en el éxito competitivo de los recursos, sus capacidades y de su interacción con la estrategia empresarial. Desde el punto de vista de los autores González, Aguilera y Rodríguez, (2011) se expone que: 'En el ámbito empresarial el análisis de la situación de competitividad es una herramienta necesaria", aunque, según González (2009), "no siempre es evaluada como una variable que resulta de la interacción de muchas variables, tanto de orden interno a la empresa como de aquellas que están presentes en el entorno empresarial'.

Aunado a lo antes expuesto, la crisis financiera internacional obligó a reflexionar sobre la viabilidad de las micro, pequeñas y medianas empresas mexicanas, reconociendo el papel que las MIPYMES, por ser un instrumento de cohesión y estabilidad social al dar oportunidades de empleo (bien sea formal o informal) a personas con o sin formación profesional, por ello el conocimiento del comportamiento de los sectores productivos en México, es útil para entender la dinámica de los factores que motivan el crecimiento en las actividades económica, un patrón de decisiones en la compañía que determinan los objetivos y metas, generando planes y políticas para el logro de los mismos y considerando los factores económicos y humanos en beneficio de los accionistas -empleados y clientes- y la comunidad ; sin embargo, en la formulación de estrategias.

En palabra de los autores Narváez y Fernández, (2008) se establece que: 'Existen estrategias genéricas en las cuales las empresas pueden conseguir ventajas competitivas mediante los costos bajos es decir que participen en el mercado con una ventaja en costos que sea atractiva a los 
clientes". En ese sentido, se analiza que una empresa es la unidad de producción económica legalmente constituida por personas y materiales, ya que una buena estrategia permite la financiación de proyectos rentablemente marginales que no pueden ser financiados como empresas de negocio único.

Asimismo, según Francés, (2010): '’El paradigma de competencia se fundamenta en cambiar la competencia entre empresas a través de una buena cooperación, el punto de partida para esta estrategia es pensar en los complementos que son necesarios". Para facilitar el desarrollo de competencias y condiciones es necesario aplicar medios, técnicas, conocimiento, inteligencia y modelos empresariales.

Por su parte, desde el punto de vista del autor Vargas, (2003), se manifiesta que: " Al cambiar el medio ambiente competitivo por el fenómeno de la turbulencia, con mayor complejidad e incertidumbre, la alta dirección de las organizaciones debe responder a crecientes demandas impuestas por las nuevas condiciones económicas y sociales". Los líderes de las empresas u organizaciones deben de responder correctamente a las nuevas condiciones económicas y sociales, mediante un proceso continuo de innovación de todas las actividades de una empresa buscando ventajas competitivas fundadas especialmente en el conocimiento de todos los miembros de la organización para poder enfrentarse a las nuevas competencias.

\section{Principios metodológicos del estudio}

Una vez expuesto el fundamento teórico esencial sobre la estrategia gerencial se declara que, esta investigación es documental, la misma se asume como una investigación confirmatoria de diseño documental, la cual se define según Hurtado de B., (1998) en (Fernández, 2007) como:

La modalidad de investigación cuyo objetivo es la verificación de datos e información exhaustiva y rigurosa, utilizando en forma precisa la documentación existente para ello. En este caso, las fuentes de información son documentos, archivos, informes, estudios, textos, memorias, anuarios, prensa, artículos, material cartográfico, entre otros. (p. 222) 
También se define como una investigación descriptiva, la cual se interesa por "especificar propiedades, características y rasgos relevantes de un tema de estudio determinado, con el objeto de analizarlo y describirlo, en el marco del cual se recolectan datos sobre diversos conceptos para describirlos”. Hernández, Fernández y Baptista (2014). En el marco de este contexto metodológico, se asume como técnica el análisis de contenido que permite estudiar la información de interés de manera sistemática y si es el objetivo facilita la descripción de contenidos por unidades de análisis y categorías.

De ello se hizo una selección de artículos con rango de publicación por año desde 2010, 2016, de cuya revisión se desprende que, de la lectura de los textos se obtienen unidades de análisis, categorías y subcategorías desde la exégesis de los categorizadores. En este caso particular, se hizo el análisis con cinco categorizadores que leyeron analíticamente los textos y derivaron de ello mediante registro y jerarquización de palabras alusivas al tema de estudio, debido a la reiteración de la aparición y el uso de términos, las unidades de análisis, las categorías y las subcategorías, lo cual permitió su análisis y las posteriores reflexiones finales. De tal suerte que, a mayor cantidad de aparición de palabras se extraen las unidades de análisis y en orden decreciente se toman las reiteraciones para las categorías y subcategorías emitidas por los autores de los textos leídos y analizados a este tenor.

\section{Análisis y Discusión de Resultados}

De la lectura y análisis se obtuvo que los autores abogan por la asunción de estrategias gerenciales de organización, tal como se evidencia en el artículo de Arce (2010) "Cómo lograr definir objetivos y estrategias empresariales" donde se observa que el autor asume la acción gerencial como la aplicación de estrategias de organización en correspondencia con los objetivos, los fines y las metas de la empresa, en pos de lograr competitividad, éxito y eficacia en la gestión gerencial. Por su parte, en el artículo de los autores: Aguilera, L. Gonzales, M y Rodríguez, R. (2011), denominado: "Estrategias empresariales para la competitividad y el crecimiento de la PYMES. Una evidencia empírica" se da cuenta de que los autores coliden con esto y además adoptan el ámbito empresarial como el contexto en el cual se establecen metas y se desarrollan acciones que impliquen el alcance del logro del éxito de la competitividad, tal como seobserva 
en el siguiente cuadro denominado matriz categorial 1 en el cuadro $\mathrm{N}^{\circ} 01$ y en la figura $\mathrm{N}^{\mathrm{o}} 01$, a saber:

Cuadro 1. Matriz Categorial 1

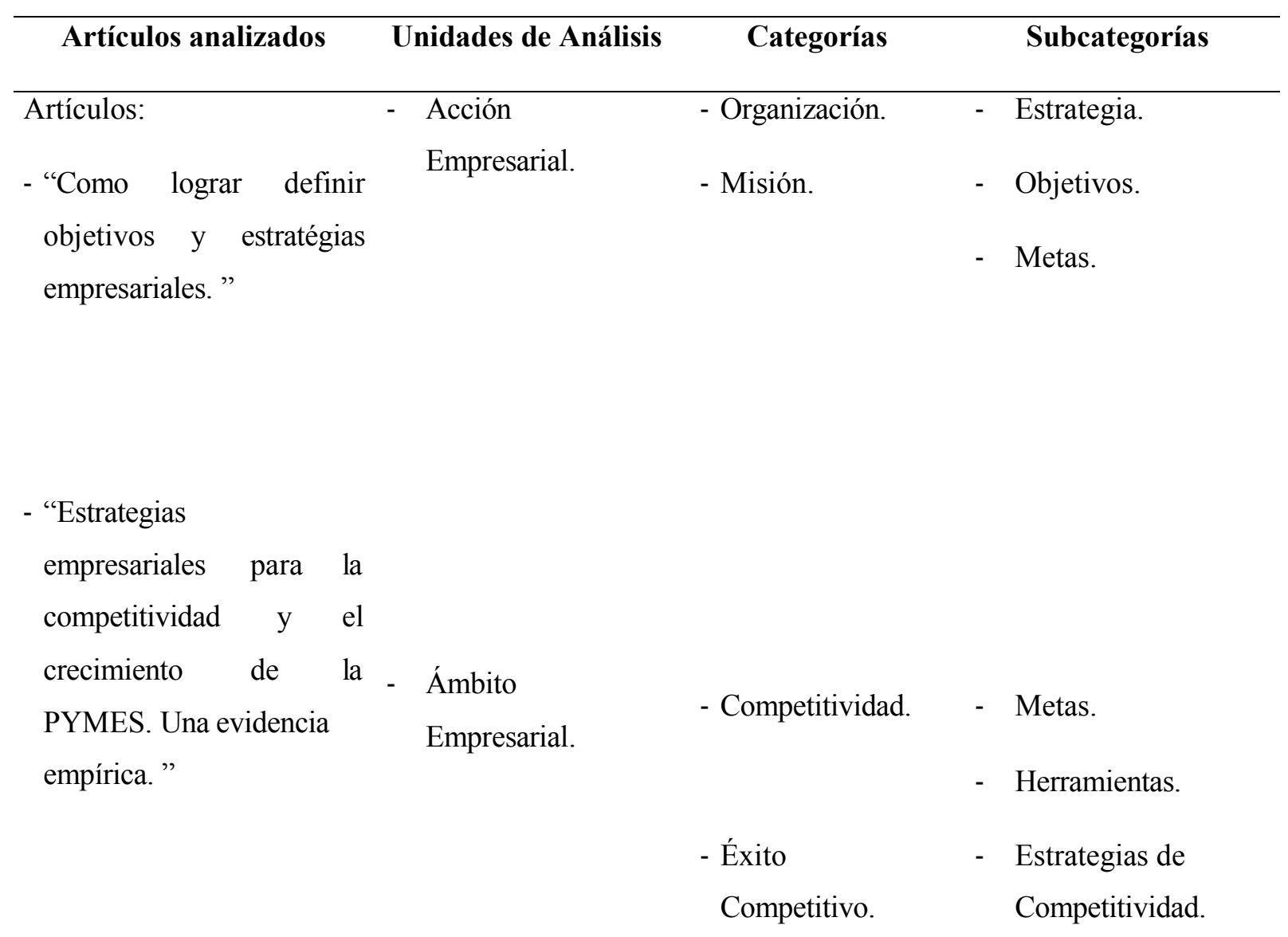

Fuente: Artículos: “Como lograr definir objetivos y estrategias empresariales.” De Arce (2010) y "Estrategias empresariales para la competitividad y el crecimiento de la PYMES. Una evidencia empírica.” De Aguilera, Gonzales, y Rodríguez, (2011),

Elaboración: propia (2019). 


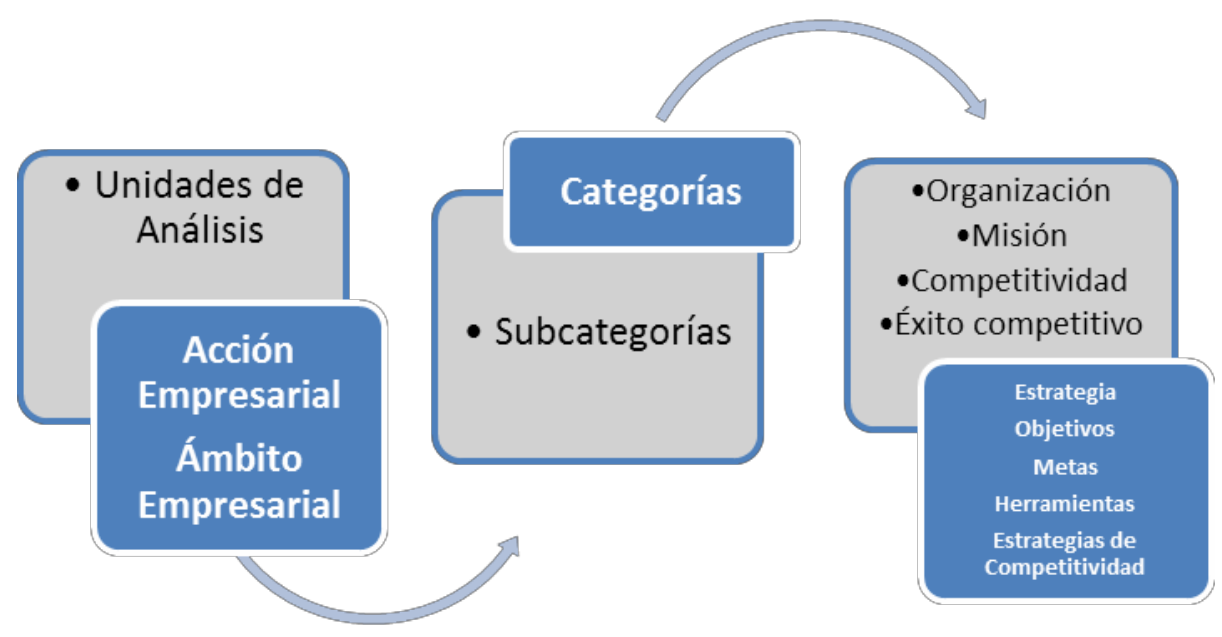

Figura Nº1 Unidades de Análisis, Categoría y Subcategorías de los Artículos: "Como lograr definir objetivos y estrategias empresariales." De Arce (2010) y "Estrategias empresariales para la competitividad y el crecimiento de la PYMES. Una evidencia empírica." De Aguilera, Gonzales, y Rodríguez, (2011)

Aunado a lo antes expuesto, en los artículos: Estrategia y competitividad empresarial: Un estudio en las Mi PyMEs de Tubasco, de Aragón, Rubio, Serna y Chablé (2010); en: "La rentabilidad económica, un factor para alcanzar la eficiencia empresarial" de Cantero, y Leyva, (2016); y en: "Estrategia empresarial e innovación tecnológica de las industrias" original de Corrêa, Sugahara, y Rodríguez, (2015) se evidencia que estos autores toman la actividad empresarial como una gestión que comporta la adecuación de la organización en dependencia de qué tipo de empresa se trata, en correspondencia de si se trata de una pequeña o mediana empresa de sus metas y objetivos que implique la asunción de la competitividad, los enfoques de metas y estrategias empresariales sustentadas en la innovación y el avance que comporta la adopción de nuevas metodologías con renovadas técnicas y métodos, que generen innovación y resultados económicos eficaces para un el logro exitoso de aumento de productividad, con calidad total, optimización de recursos y búsqueda de diversas fuentes de financiamiento. Los resultados del análisis de estos artículos, se resumen en la Matriz Categorial 2 y en la figura $\mathrm{N}^{\circ} 02$, que a continuación se presentan: 
Cuadro 2. Matriz categorial 2

\begin{tabular}{llll}
\hline \multicolumn{1}{c}{ Artículos analizados } & Unidades de Análisis & \multicolumn{1}{c}{ Categorías } & Subcategorías \\
\hline & & & Innovación \\
"Estrategia y competitividad & Pequeñas y medianas & Éxito Competitivo. & Tecnología \\
$\begin{array}{l}\text { empresarial: Un estudio en las } \\
\text { Mi PyMEs de Tubasco." }\end{array}$ & empresas & Metas & Estrategias \\
& & & Competitivas.
\end{tabular}

"La rentabilidad económica, un factor para alcanzar la eficiencia empresarial."

Empresa

"Estrategia empresarial e innovación tecnológica de las industrias"

Procesos de

Financiamiento. Eficiencia

Optimización de recursos.
Procesos

Economía exitosa

\section{Organización}

Competitividad

Metas
Estrategia

Tecnología

Innovación.

Fuente: Artículos: "Estrategia y competitividad empresarial: Un estudio en las Mi PyMEs de Tubasco", de Aragón, Rubio, Serna y Chablé (2010); "La rentabilidad económica, un factor para alcanzar la eficiencia empresarial” de Cantero, y Leyva, (2016);

"Estrategia empresarial e innovación tecnológica de las industrias” original de Corrêa, Sugahara, y Rodríguez, (2015).

Elaboración: Propia, 2019. 


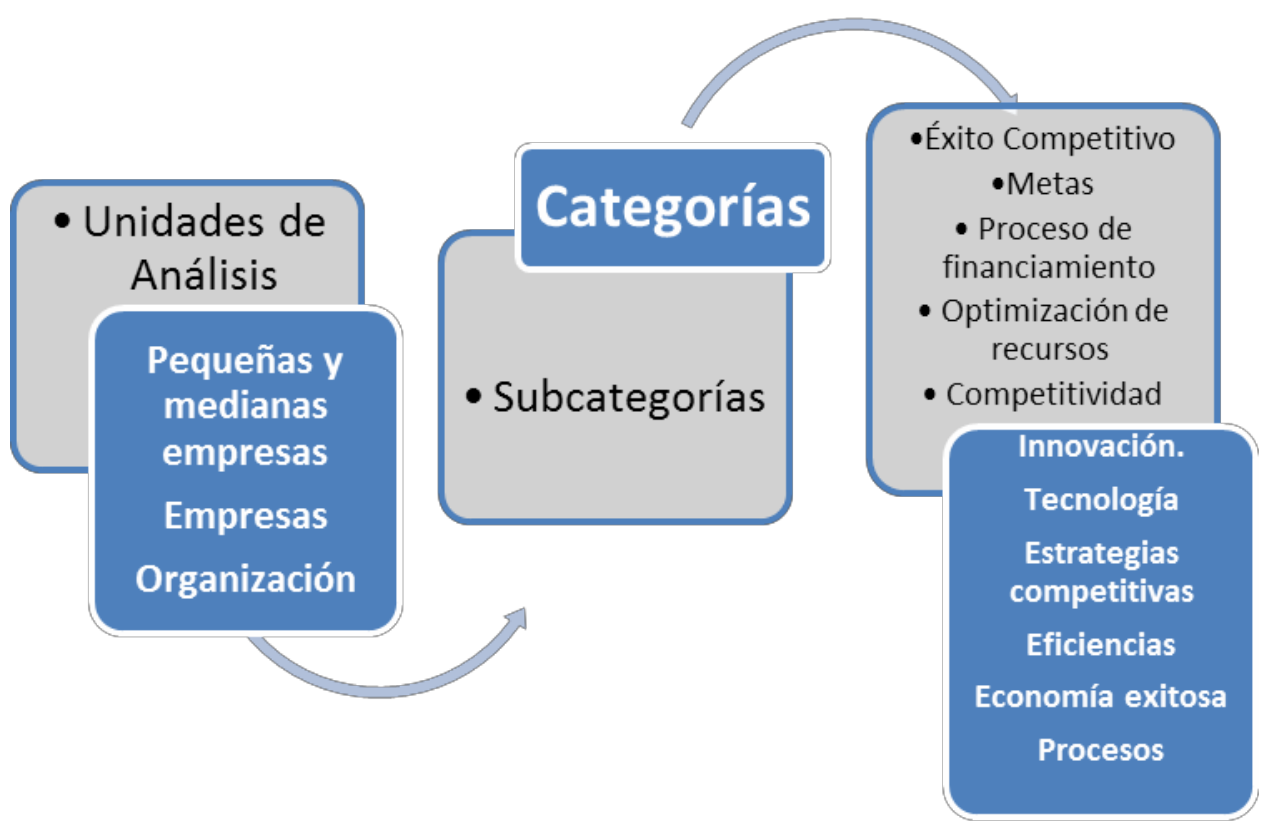

Figura No 02 Unidades de Análisis, Categoría y Subcategorías de los artículos: "Estrategia y competitividad empresarial: Un estudio en las Mi PyMEs de Tubasco", de Aragón, Rubio, Serna y Chablé (2010); "La rentabilidad económica, un factor para alcanzar la eficiencia empresarial" de Cantero, y Leyva, (2016); "Estrategia empresarial e innovación tecnológica de las industrias” original de Corrêa, Sugahara, y Rodríguez, (2015)

\section{Conclusiones}

De los artículos revisados se puede decir que se asumen las Estrategias Gerenciales como aquellas acciones que se adoptan y efectúan en el desarrollo de una actividad empresarial, con el fin de obtener resultados positivos, teniendo guías en un grupo para desempeñar las diferentes actividades con liderazgo del gerente. Se observó que las empresas deben poseer también niveles de planeación estratégica, los mismos que suelen variar dependiendo de la complejidad organizacional, aquellos niveles presentan siempre una diferente aplicación en las pequeñas y medianas empresas, en comparación con las grandes que poseen una estructura organizativa particular y suelen estar divididas por departamentos.

De ello ha dado cuenta este artículo toda vez que, al analizar los trabajos de De Arce (2010); de Aguilera, Gonzales, y Rodríguez, (2011), Aragón, Rubio, Serna y Chablé (2010); de Cantero, y 
Leyva, (2016); y de Corrêa, Sugahara, y Rodríguez, (2015) surgieron como unidades de análisis, las que siguen a continuación: Acción Empresarial, Ámbito Empresarial, Pequeñas y medianas empresas, Empresa y Organización, en ellas se imbrican las siguientes categorías: Organización, misión, competitividad, éxito competitivo, metas, proceso de financiamiento, optimización de recursos, y en éstas últimas se insertan las siguientes subcategorías: Estrategia, objetivos, metas, herramientas, estrategias de competitividad, innovación, tecnología, eficiencia, procesos, economía exitosa.

Estos resultados evidencian que los autores de los artículos analizados, proponen la asunción de que, en los ámbitos de investigación de la Carrera Administración de Empresas, los autores latinoamericanos abogan por la asunción de las Estrategias Empresariales que se sustenten en la tecnología para generar innovación y eficacia económica. Esto aunado a la planificación de la organización con arreglo a las metas institucionales, la optimización en el manejo de los recursos, la formación del talento, la competitividad, y el éxito económico. Por todo ello se concluye que, La estrategia es el camino que las organizaciones siguen para alcanzar un objetivo, para mejorar el desempeño dentro de la organización, mientras que el proceso de toma de decisiones estratégicas es el que sirve para corregir el rumbo de las organizaciones, hacia un objetivo más deseable.

\section{Referencias Bibliográficas}

Aguilera, L. Gonzales, M y Rodríguez, R. (2011). Estrategias empresariales para la competitividad y el crecimiento de la PYMES. Una evidencia empírica. Investigación y ciencia, vol.19 (53), pp.39-48.

Alburquerque, F. (1995). Competitividad internacional, estrategia empresarial y papel de las regiones. Revista EURE, vol. (63), pp. 41.

Aragón, A y Rubio, M., Serna y Chablé (2010) Estrategia y competitividad empresarial: Un estudio en las Mi PyMEs de Tubasco. Revista de Tubasco, vol.47, pp. 4-12.

Acevedo, P. (2009). Estudio del estado del arte de las competencias en América Latina, Europa, México y su relación con el Nuevo Modelo Educativo y la Escuela Superior de Comercio y Administración Unidad Tepepan. $9^{\circ}$ Congreso Internacional de la Universidad. (1) 3 - 5. 
Ansoff, H. I. The changing shape of strategic problem. In: SHENDEL and HOFER (Eds.). Strategic management: a new view of business policy and planning. Little Brown, 30-52, 1979.

Arce, L. (2010). Como lograr definir objetivos y estrategias empresariales. Perspectivas, vol. (25), pp. 191-201.

Bayón, F., Cubeiro, J., Romo, M. (2006) Coaching realmente. Madrid: Pearson Prentice Hall.

Bohórquez, L. E. (2013). La organización empresarial como sistema adaptativo complejo. Estudios Gerenciales, vol. (29), pp. 258-265

Cantero, H y Leyva, E. (2016). La rentabilidad económica, un factor para alcanzar la eficiencia empresarial. Ciencias Holguín, vol.22 (4), pp1-17.

Chávez, H. (2012). La gestión por competencias y ejercicio del coaching empresarial, dos estrategias internas para la organización. Pensamiento \& amp; Gestión, vol. (33), pp. 140161.

Corrêa, A., Sugahara, R., Rodríguez, J. (2015) Eduardo estrategia empresarial e innovación tecnológica de las industrias Brasileñas Invenio, Redalyc, vol. 18, núm. 35, pp. 65-84

Drucker, P. (1986) The New Realities: In Govern-ment and Politics, in Economics and Business, in Society and World View, Harper \& Row, New York, 1989.

Fernández, I. (2007) Diccionario de Investigación. Una Comprensión Holística. Segunda Edición. Quirón, Sypal. Caracas, Venezuela

Francés, A. (2010) ¿Cómo mejorar la competitividad de las empresas nacionales? Debates IESA, vol. $7 \mathrm{~N}^{\mathrm{o}}$ 4, abril-junio 2010. Ediciones IESA, Caracas.

Ghani, I., Ariffin, Z., Zainuddin, Y. (2008). Competency based Career Develop Competency based Career Development and Performance Management Practices and Service Quality in Malaysian Public Organizations. Academic Journal, 4 (2), 235 - 241.

González, G. (2009) Ganancias de competividad: Un enfoque agregado y de largo plazo. Análisis Económico. XXIV (57) pp. 81-104. 2009. 
Henderson, B. (1979). Henderson on Corporate Strategy. Abt Books, Cambridge, Ma-

Hernández, N. (2015). E-learning en la estrategia organizativa. Indivisa. Boletín de Estudios e Investigación, vol. (15), pp. 11-23.

Hernández, S., Fernández, C. y Baptista, P. (2014) Metodología de la investigación. México DF, México: Editorial Mc Graw Hill.

Hurtado de B., J. (1998) Metodología de la Investigación Holística. Fundación Sypal. Fundacite Anzoátegui, Caracas, Venezuela

Koontz, H \& Weihrich, H. (2010). Administración una perspectiva global. Educación, vol. 14, pp. 4-10.

Lazzati, S. (2010). Estrategias para el desarrollo de competencias conductuales. Talento Humano, $14,32-33$.

Lozada, Gutiérrez y Arias (2010) Perspectivas Predominantes sobre Estrategias Gerenciales en Colombia. Revista Perfil de Coyuntura Económica ISSN 1657-4214. Universidad de Antoquia, Medellín, Colombia.

Leal, M. y Cefalá, Y. (2003) Estrategias Crecimiento Empresarial aplicadas por Hipermercados. Revista Venezolana de Gerencia (RVG). Año 8, № 24, 2003, 607-621. Universidad del Zulia, LUZ ISSN 1315-9984

Mudler, M. (2007).Competencia: la esencia y la utilización del concepto en la formación profesional inicial y permanente. Revista Europea de Formación Empresarial (Universidad de Wageningen), 40, 5 - 24.

Narváez, M. y Fernández, G. (2008) Estrategias Competitivas para fortalecer sectores de actividad empresarial en el mercado global. Revista Venezolana de Gerencia. V. 13 n 42, Maracaibo, junio 2008

Páez, I. (2006). Competencias para el liderazgo gerencial, Journal Sotavento de la facultad de Administración de Empresas, Universidad Externado de Colombia, Vol. 1, pp. 78 - 97.

Palomo, M. (2010). Gestión por competencias y talento. Madrid: ESIC. 
Romero, M. (2002) Tendencias organizacionales de las Empresas. Revista de Gerencia (RVG) Año 7. Nº 19, 2002, 355-374 Universidad del Zulia (LUZ) · ISSN 1315-9984

Santos, J. (2001). El desarrollo de un modelo de gestión por competencias basado en directorios sectoriales. Boletín de Estudios Económicos, 174, 537 - 574.

Suárez, J \& Ibarra, S. (2008). La teoría de los recursos y las capacidades. Un enfoque actual de la estrategia empresarial. Departamento de ingeniero industrial, pp. 1-27.

Vargas, J. (2003) La Organización y la estrategia: preferencias del estratega o imperativo para el éxito. RAM. Revista de Administración Mackenzie. vol. 4, núm. 2, 2003, pp. 88-116 Universidad de Presbiteriana Mackenzie São Paulo, Brasil 